\title{
O efeito assimétrico na volatilidade dos preços do etanol no Estado de São Paulo: uma aplicação do modelo Asymmetric power autoregressive conditional heteroskedasticity
}

O presente artigo examina a volatilidade dos retornos semanais dos preços do etanol hidratado por meio do modelo de variância condicional, também chamado heteroscedástico. A análise compreende o período de 29 de novembro de 2002 a 20 de novembro de 2020 . Os resultados empíricos demonstraram as reações de persistência e assimetria na variância dos respectivos retornos, ou seja, boas e más notícias impactam diferentemente sobre a volatilidade dos retornos de acordo com o modelo ARMA(1,1)- APARCH $(1,1)$, com distribuição generalizada de erro, do ponto de vista da realização de previsões.

Palavras-chave: Efeito alavancagem; Volatilidade de preços; APARCH.

\section{The asymmetric effect on the volatility of ethanol prices in the State of São Paulo: an application of the Asymmetric power autoregressive conditional heteroskedasticity model}

\begin{abstract}
This article examines the volatility of weekly returns on hydrous ethanol prices using the conditional variance model, also called heteroskedasticity. The analysis covers the period from November 29, 2002 to November 20, 2020. The empirical results demonstrated the reactions of persistence and asymmetry in the variance of the respective returns, that is, good and bad news impact differently on the volatility of the returns according with the ARMA (1.1) - APARCH (1.1) model, with generalized error distribution, from the point of view of making forecasts.
\end{abstract}

Keywords: Leverage effect; Price volatility; APARCH.

Topic: Finanças Empresariais

Reviewed anonymously in the process of blind peer.
Received: 07/04/2021

Approved: 04/06/2021

Carlos Alberto Gonçalves da Silva

Universidade do Estado do Rio de Janeiro, Brasil

http://lattes.cnpq.br/4301394305558781

http://orcid.org/0000-0002-6827-5073

ca7gon@gmail.com

Referencing this:

SILVA, C. A. G.. O efeito assimétrico na volatilidade dos preços do etanol no Estado de São Paulo: uma aplicação do modelo Asymmetric power autoregressive conditional heteroskedasticity. Revista Brasileira de Administração Científica, v.12, n.2, p.1-12, 2021. DOI: http://doi.org/10.6008/CBPC2179-684X.2021.002.0001 
O efeito assimétrico na volatilidade dos preços do etanol no Estado de São Paulo: uma aplicação do modelo Asymmetric power autoregressive

\section{INTRODUÇÃO}

Engle (1982) em seu trabalho seminal teve como objetivo estimar a variância da inflação no Reino Unido, a partir de informações e dados dos anos 70 . O resultado de seu trabalho evidenciou a existência de variância condicional nessa série de retornos, projetando posteriormente uma grandiosidade de trabalhos sobre o modelo ARCH. Em sua segunda publicação, Engle (1982) se utilizou da modelagem ARCH para a definição do risco de uma carteira de investimentos, assumindo que o mesmo seguia um processo de variância condicional. Em 2001, na publicação "The use of ARCH/GARCH models in applied econometrics", Engle (1982), através dos modelos ARCH e GARCH comprovou que em diversas séries temporais financeiros, as várias extensões desses modelos podiam ser testadas e exibidas, por exemplo, na precificação de ativos e análises de carteiras, revalidando e corroborando a aplicação do modelo ARCH em finanças.

A generalização do modelo ARCH foi elaborada por Bollerslev (1986) quando apresentou o modelo GARCH. O autor propôs a inserção de variância de defasagens no modelo, uma espécie de instrumento adaptativo. No modelo $\mathrm{ARCH}$, a variância condicional é função das variâncias amostrais. A apresentação empírica do modelo foi baseada na taxa de inflação americana e o modelo foi amplamente aceito e utilizado, inclusive por Engle (1982).

Nelson (1991) estruturou a partir dos modelos ARCH/GARCH uma nova abordagem- "Conditional Heteroskedasticity in Asset Returns: A New Approach" - no qual o autor adiciona um complemento exponencial ao modelo ARCH, derivando-o ao modelo Exponencial Generalized Autoregressive Conditional Heteroscedasticity (EGARCH), reiterando ainda, perspectivas para mais pesquisas.

Zakoian (1994) considerou uma alteração no modelo clássico ARCH proposto por Engle (1982). No seu trabalho foi apresentado o modelo TARCH, onde o desvio padrão condicional é uma função linear por partes dos valores passados do ruído branco. Esta forma específica permitiu que diferentes reações da volatilidade de sinais diferentes fossem captadas pelo modelo. A seguir realiza-se um breve comentário de alguns trabalhos utilizando modelos condicionalmente heteroscedásticos.

Mota et al. (2004), compararam modelos da família GARCH com estimadores alternativos baseados em cotações de abertura, fechamento, máximo e mínimo. Os resultados indicaram que os estimadores alternativos são precisos quanto aos modelos do tipo GARCH.

Morais et al. (1999) apresentaram modelos da família GARCH que captam diferentes efeitos observados em séries financeiras, tais como a aglomeração da variância, o efeito "leverage" e a persistência na volatilidade. Neste estudo é comparada a estimativa da volatilidade do índice Bovespa obtida por processos determinísticos e estocásticos, abrangendo três períodos conturbados: a crise do México, a crise Asiática e a moratória Russa. Os resultados do estudo mostraram que ambos os processos conseguem prever a volatilidade.

Costa Junior et al. (1999) examinaram a influência de eventos sobre a volatilidade nos mercados de ações da América Latina, utilizando o modelo GJR-GARCH (1,1)-M. O estudo utiliza índices diários dos mercados de ações e abrange um período compreendido entre janeiro de 1995 e dezembro de 1998. Os 
resultados obtidos sugerem que a influência dos eventos negativos é superior à dos eventos positivos na maioria dos países analisados.

Freitas et al. (2015) analisaram a persistência, a alavancagem e a variância condicional dos retornos de commodities (etanol, açúcar, soja, milho, café, algodão, arroz, trigo, frango, boi gordo e bezerro), utilizando o modelo APARCH. Os resultados mostraram que a variância condicional foi assimétrica apenas nos retornos do etanol, do café, do algodão, do boi gordo e do bezerro.

Em relação à aplicação de séries financeiras, autores brasileiros e internacionais desenvolveram trabalhos, utilizando os modelos condicionalmente heteroscedásticos, pode-se citar Duarte et al. (1996), Almeida et al. (1999), Issler (1999), Baidya et al. (1999), Engle et al. (1986), Bollerslev et al. (1992), Silva (2006), Araujo et al. (2019), Nugroho et al. (2017) e Laurent (2004).

Neste estudo o objetivo é avaliar empiricamente o processo de volatilidade dos retornos dos preços do etanol hidratado, examinando-se dois fatores determinantes, a persistência de choques e assimetria da volatilidade, por meio da aplicação do modelo Asymmetric Power autoregressive conditional heteroskedasticity (APARCH).

\section{METODOLOGIA}

\section{Testes de estacionariedade}

Para testar a estacionariedade das séries, será utilizado neste trabalho, o teste ADF (Dickey - Fuller Aumentado) (DICKEY et al. 1979), no sentido de verificar a existência ou não de raízes unitárias nas séries temporais. O teste de Dickey-Fuller Aumentado (ADF) consiste na estimação da seguinte equação por Mínimos Quadrados Ordinários (MQO):

$$
\Delta Y_{t}=\alpha+\beta t+\gamma Y_{t-1}+\sum_{i=1}^{p} \delta_{i} \Delta Y_{t-1}+\varepsilon_{t}(1)
$$

onde $\Delta Y_{t}$ é o operador de diferenças $\left(Y_{t}-Y_{t-1}\right)$, $\alpha$ é uma constante, $\beta t$ é a componente de tendência do modelo, $\gamma$ é o coeficiente que permite testar a estacionariedade (se ${ }_{\gamma}=0, Y$ tem uma raiz unitária), $p$ é o número de termos defasados a incluir no modelo e $\varepsilon_{t}$ é o termo de erro aleatório ou perturbação estocástica.

O teste Phillips et al. (1988) também é usado para investigar a presença de raiz unitária da série. 0 que difere entre os dois testes é o fato de que o teste Phillips-Perron garante que os resíduos são não correlacionados e possuem variância constante. Ao contrário do teste Dickey-Fuller Aumentado, o teste de Phillips-Perron não inclui termos de diferenças defasadas, mas pode também incluir termos de tendência e um intercepto.

O teste KPSS (KWIATKOWSKI et al., 1992), surgiu como uma forma de complementar a análise dos testes de raiz unitária tradicional, como testes ADF e PP. Ao contrário dos testes ADF e PP, o teste KPSS, considera como hipótese nula que a série é estacionária, ou estacionária em torno de uma tendência determinística, contra a hipótese alternativa que um caminho aleatório está presente. 
Teste de normalidade da série: Jarque Bera (JB) (JARQUE et al., 1987)

O teste de normalidade Jarque-Bera $(J B)$ é baseado nas diferenças entre os coeficientes de assimetria e curtose da série e os da lei normal, servindo para testar a hipótese nula de que a amostra foi extraída de uma distribuição normal. Para a realização deste teste, calcula-se, primeiramente a assimetria e a curtose dos resíduos e utiliza-se a estatística de teste:

$$
J B=n\left[\frac{S^{2}}{6}+\frac{(C-3)^{2}}{24}\right]
$$

Onde $J B$ é o teste Jarque-Bera, $S$ é o coeficiente de assimetria das observações, $C$ é o coeficiente de curtose das observações e $n$ é o número de observações.

Sob a hipótese nula de normalidade, a estatística $J B$ segue a distribuição qui-quadrado com dois graus de liberdade. Se o valor de JB for muito baixo, a hipótese nula de normalidade da distribuição dos erros aleatórios não pode ser rejeitada. Se o valor de JB for muito alto, rejeita-se a hipótese de que os resíduos ou erros aleatórios se comportam como uma distribuição normal. Se o valor $p$ da estatística qui-quadrado calculada for suficientemente baixo, pode-se rejeitar a hipótese de que os resíduos têm distribuição normal. Se o valor $p$ for alto, se aceita a hipótese de normalidade.

\section{Teste de linearidade (BDS)}

Uma vez detectada que a distribuição não é normal, faz-se necessário testar o modelo para linearidade. Este teste foi desenvolvido por Brock et al. (1987), usado para testar se as variáveis aleatórias que compõem uma série são independentes e identicamente distribuídas (IID), ou seja, pode verificar várias situações em que as variáveis não são IID, tais como não estacionariedade, não linearidade e caos determinístico. $O$ teste se baseia no conceito de correlação espacial da teoria do caos e segundo os autores a estatística BDS é formulada como:

$$
W_{m}^{n}(\varepsilon)=\frac{\sqrt{N}\left(C_{m}^{n}(\varepsilon)-\left(C_{1}^{n}(\varepsilon)\right)^{m}\right.}{\sigma_{m}(\varepsilon)}
$$

Onde $W_{m}^{n}(\varepsilon)$ converge para uma distribuição normal $\mathrm{N}(0,1)$, à medida que $\mathrm{n}$ tende para infinito. Assim sendo, os testes de hipóteses são:

$H_{0}$ : a série segue um processo iid (independente e identicamente distribuído).

$$
H_{1} \text { : a série não segue um processo iid. }
$$

\section{Modelos ARMA}

Modelos univariados são aqueles em que os valores correntes de uma série de tempo são relacionados apenas com seus próprios valores passados ou com os valores correntes e passados dos resíduos da série temporal.

A combinação dos modelos autorregressivos (AR) e de médias móveis (MA) resulta no modelo denominado ARMA. Assim, tratando-se de uma série temporal $Y_{t}$ e considerando-se suas primeiras 
O efeito assimétrico na volatilidade dos preços do etanol no Estado de São Paulo: uma aplicação do modelo Asymmetric power autoregressive

diferenças $Y_{t}=Y_{t}-Y_{t-1}$, com $p$ defasagens para a variável e $q$ para os erros aleatórios, pode-se expressar o modelo $\operatorname{ARMA}(p, q)$ da seguinte maneira:

$$
Y_{t}=\mu+\phi_{1} Y_{t-1}+\phi_{2} Y_{t-2}+\ldots \ldots+\phi_{p} Y_{t-p}+\varepsilon_{t}-\theta_{1} \varepsilon_{t-1}-\theta_{2} \varepsilon_{t-2}-\ldots \ldots . .-\theta_{q} \varepsilon_{t-q}
$$

O modelo ARIMA ( $p, d, q)$, onde $p$ refere-se ao número de defasagens da série, $d$ é a ordem de integração e $q$ é o número de defasagens dos erros aleatórios. Portanto, utiliza-se os procedimentos sugeridos por Box et al. (1976), cujas etapas são as seguintes: a) Identificação - Determina-se os valores apropriados de $p, d$ e $q$ e utiliza-se um correlograma apresentando as funções de autocorrelação parcial (FACP) e de autocorrelação simples (FAC), podendo determinar a ordem apropriada dos componentes AR e MA; b) Estimação - esta etapa vem após a identificação e consiste em estimar os parâmetros autorregressivos e de médias móveis. Verifica-se a significância estatística dos parâmetros sugeridos para o modelo; c) Verificação - Consiste em verificar se o modelo estimado se ajusta adequadamente à série ou não e se os resíduos calculados apresentam ou não problemas de autocorrelação, bem como existência de heterocedasticidade. Os critérios de informação de Akaike e de Schwarz ajudam neste diagnóstico, bem como a utilização da estatística $Q$ de Ljung-Box. $O$ teste $Q$ é usado para testar se um conjunto de autocorrelações de resíduos é ou não estatisticamente diferente de zero. A estatística $Q$ conterá aproximadamente uma distribuição $X^{2}$ com $K-p-q$ graus de liberdade; d) Previsão - O modelo ARIMA estimado pode ser utilizado nas previsões, tornando-se possível calcular previsões de $Y_{t}$ para $n$ períodos seguintes, desde que sejam conhecidos $Y_{t-1}$ e $Y_{t}$.

\section{O modelo APARCH}

Ao longo dos anos, diversos modelos foram sendo desenvolvidos no sentido de incorporar diferentes comportamentos das séries de dados financeiros: GARCH, EGARCH, GJR-GARCH e TARCH. Neste estudo, optou-se por utilizar o modelo APARCH devido ao fato de este ser capaz de se comportar como os outros sete modelos da família ARCH.

O modelo APARCH (Asymetric Power Autorregressive Conditional Heteroskedasticity) proposto por Ding et al. (1993), oferece uma forma geral em que a potência da equação da variância condicional é estimada, bem como os parâmetros tradicionais como alpha e beta, pode ser representado por:

$$
\begin{aligned}
& \sigma_{t}^{\delta}=\omega+\sum_{i=1}^{p} \alpha_{i}\left(\left|\varepsilon_{t-i}\right|-\gamma_{i} \varepsilon_{t-i}\right)^{\delta}+\sum_{j=1}^{q} \beta_{j} \sigma_{t-j}^{\delta} \\
& \quad \text { onde } \omega ; \alpha_{i} \geq 0 \text {, para } i=1, \ldots \ldots, p ; \beta_{j} \geq 0 \text {, para } j=1, \ldots \ldots, q ; \delta \geq 0 \text { e }-1<\gamma_{i}<1 .
\end{aligned}
$$

Este modelo pode detectar impactos assimétricos de choques sobre a volatilidade. Se $\gamma_{i}>0$, verifica-se o efeito alavancagem, isto é, choques negativos tem um impacto maior sobre a volatilidade da série que choques positivos de um dia à frente. Se $\gamma_{i}<0$, indica que choques positivos tem maior impacto sobre a volatilidade que choques negativos. $\mathrm{O}$ parâmetro $\delta$ permite determinar outras potências para a 
equação da variância condicional, através de uma transformação Box-Cox do $\sigma_{t}$, à qual pode estimar várias potências para o desvio padrão condicional, como acontece como o modelo $\mathrm{GARCH}$, utilizando-se apenas a potência quadrática.

Charles (2010) confirma que o modelo APARCH foi considerado relevante em várias aplicações. Segundo Laurent (2004) esse modelo é um dos mais importantes da família ARCH, que abrange ao menos sete modelos ARCH como seguem:

- ARCH de Engle (1982), quando $\delta=2, \gamma_{i}=0$ e $\beta_{j}=0$;

- GARCH de Bollerslev (1986), quando $\delta=2, \gamma_{i}=0$;

- ARCH não-linear de Higgins et al. (1992), quando $\gamma_{i}=0$ e $\beta_{j}=0$;

- GARCH de Taylor (1986) e Schwert (1990), quando $\delta=1, \gamma_{i}=0$;

- TARCH de Zakoian (1994), quando $\delta=1$ e $\beta_{j}=0$;

- Log-ARCH de Geweke (1986) e Pentula (1986), quando $\delta \rightarrow 0$;

- GJR-GARCH de Glosten-Jagannathan-Runkle (GLOSTEN et al., 1993), quando $\delta=2$.

\section{Distribuições de erros}

Para o modelo APARCH, serão ajustadas as distribuições Gaussiana (Normal), $t$-Student e Generalized Error Distribuition (GED), descritas a seguir pela função log-verossimilhança.

\section{Distribuição normal}

Balakrishnan et al. (2003) mostram que a função densidade da probabilidade normal é a distribuição mais utilizada como parâmetro de comparação em aplicações estatísticas (análise de regressão, séries temporais, etc.). Assim a distribuição normal é definida como:

$$
f(x)=\frac{1}{\sqrt{2 \pi \sigma}} \exp \left\{-\frac{1}{2}\left(\frac{x-\mu}{\sigma}\right)^{2}\right\}
$$

onde $\mu$ a média e $\sigma$, o desvio padrão da variável aleatória $x$.

Pode-se dizer que uma amostra obedece a uma distribuição Log-normal se os logaritmos de seus valores apresentam a uma distribuição normal, ou seja, representada pela seguinte maneira:

$$
L_{\text {normal }}(x)=\frac{1}{x \sigma \sqrt{2 \pi}} \exp \left(\frac{-\ln (x)-\mu}{2 \sigma}\right) \quad \text { para } x \succ 0,
$$

onde $\mu$ é a média dos logaritmos da variável $x$, e $\sigma$ o desvio padrão dos logaritmos da variável aleatória $x$.

\section{Distribuição t- Student}

Hsieh (1989) e Baillie et al. (1989) mostraram que a distribuição $t$-Student pode capturar desempenho dos log-retornos em séries temporais. Assim, a função densidade da probabilidade pode ser descrita como: 


$$
\begin{aligned}
& L_{\text {student }-t}=\ln \left[\Gamma\left(\frac{v+1}{2}\right)\right]-\ln \left[\Gamma\left(\frac{v}{2}\right)\right]-0,5 \ln [\pi(v-2)] \\
& -0,5 \sum_{t=1}^{n}\left[\ln \sigma_{t}^{2}+(1+v) \ln \left(1+\frac{\varepsilon_{t}^{2}}{v-2}\right)\right]
\end{aligned}
$$

em que $\Gamma($.) é a função gama, $v$ corresponde aos graus de liberdade. Tem-se que $v>2$, se $v \rightarrow \infty$, a distribuição $t$ student converge para uma distribuição normal.

\section{GED (distribuição generalizada de erro)}

A distribuição (GED) foi introduzida por Subbotin (1923) e sendo inicialmente utilizada por Box et al. (1973), e posteriormente por Nelson (1991). A função densidade de probabilidade GED é expressa como:

$$
L_{G E D}=\sum_{t=1}^{n}\left[\ln \left(v / \lambda_{v}\right)-0,5\left|\frac{\varepsilon_{t}}{\lambda_{v}}\right|^{v}-\left(1+v^{-1}\right) \ln (2)-\ln \Gamma(1 / v)-0,5 \ln \left(\sigma_{t}^{2}\right)\right],
$$

$$
\text { onde } v \text { é o grau de liberdade e } \Gamma(.) \text { é a função gama, e } \lambda_{v} \equiv \sqrt{\frac{\Gamma\left(\frac{1}{v}\right) 2^{(-2 / v)}}{\Gamma\left(\frac{3}{v}\right)}}
$$

Essa distribuição generaliza a distribuição Normal podendo ter caudas mais leves $(k>2)$ ou mais pesadas $(k<2)$ do que a Normal Padrão $(N(0,1))$ e se $k=2$ obtemos a distribuição Normal.

Para verificar se o modelo considerado no estudo está bem especificado, as 'inovações' padronizadas devem formar uma sequência de variáveis aleatórias i.i.d., em que o efeito da heteroscedasticidade tenha sido eliminado. Como mencionado por Tsay (2010), utilizou-se o teste de Ljung-Box para verificar se os resíduos (inovações) padronizados são i.i.d., comprovando que a função para média está adequada, e o multiplicador de Lagrange (LM) ou o Ljung-Box dos resíduos padronizados ao quadrado para testar se o efeito ARCH foi efetivamente controlado, ou seja, que o modelo da variância condicional é adequado.

\section{Dados}

Os dados utilizados neste estudo se referem aos preços semanais do etanol hidratado, compreendendo o período de 29/11/2002 a 20/11/2020, num total de 939 observações. Os dados foram obtidos do site do Centro de Estudos Avançados em Economia Aplicada (CEPEA-ESALQ/USP). O software utilizado para se estimar o modelo de análise foi Eviews 9.0.

\section{RESULTADOS E DISCUSSÃO}

Os retornos diários foram calculados através da fórmula: $r_{t}=\ln \left(P_{t}\right)-\ln \left(P_{t-1}\right)$. Sendo que $P_{t}$ representa o preço do etanol hidratado no dia t e $P_{t-1}$ o preço do etanol hidratado no dia anterior (t-1). As Figuras 1 e 2 mostram o comportamento das séries de cotações dos preços e retornos semanais do etanol no período considerado. 


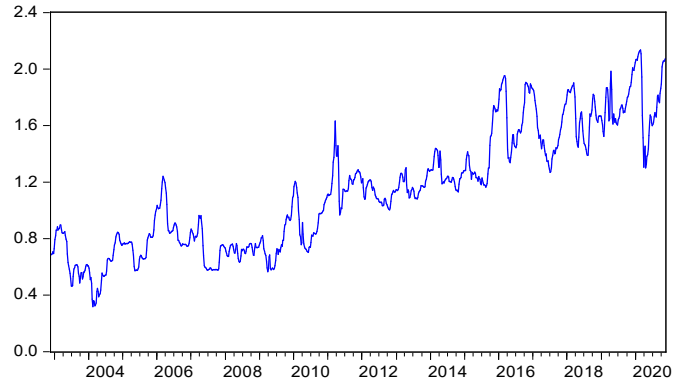

Figura 1: Cotações semanais dos preços do etanol hidratado.

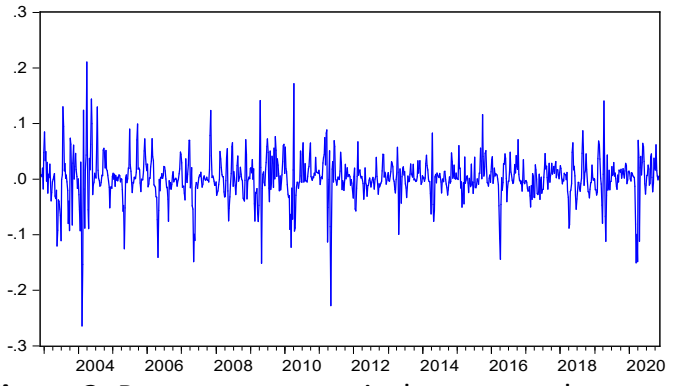

Figura 2: Retornos semanais dos preços do etanol hidratado.

Na inspeção visual da Figura 2, dentro do período de análise, nota-se uma acentuada volatilidade nos retornos, com a presença de outliers. Dessa forma foi preciso testar a normalidade e estacionariedade da série dos retornos diários dos preços do etanol hidratado para aplicação do modelo heteroscedástico.

Algumas estatísticas descritivas básicas são indicadas no gráfico 1. Observa-se que os retornos diários dos preços do etanol apresentam uma distribuição leptocúrtica devido ao excesso de curtose $(9,669783)$ em relação à distribuição normal $(3,0)$. A estatística de Jarque-Bera indicou a rejeição da normalidade da distribuição da série, com $p$-valor igual a zero.

Pela análise dos resultados, constata-se que tanto a média $(0,001188)$ quanto a mediana $(0,001461)$ apresentaram valores próximos de zero. A variação entre o valor mínimo $(-0,264)$ e o valor de máximo $(0,210)$ da série, pode ser explicada devido algumas oscilações significativas nos retornos dos preços do etanol. 0 baixo valor do desvio-padrão $(0,0392)$ indica que, de maneira geral, as altas variações na série ocorreram em poucas ocasiões, ou seja, em períodos de picos positivos e negativos.
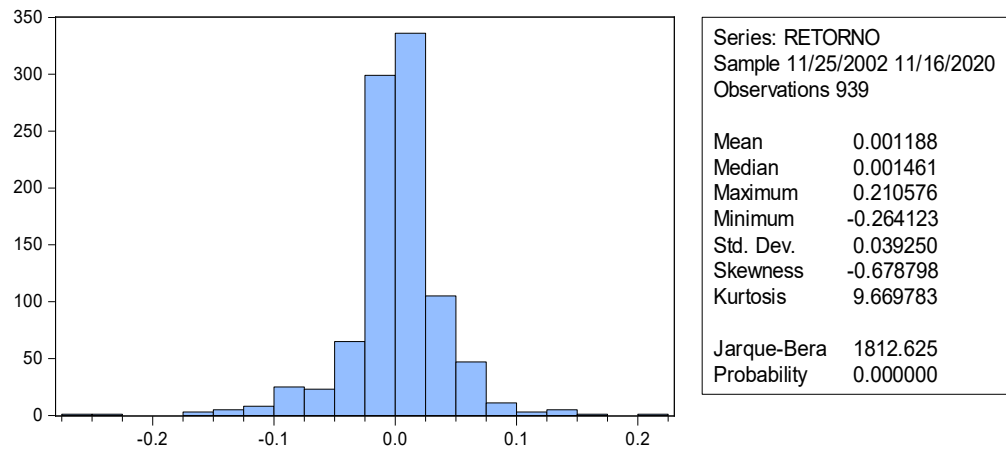

Gráfico 1: Sumário estatístico dos retornos dos preços do etanol hidratado no Estado de São Paulo.

O Q-Q Plot representa um dos métodos gráficos mais utilizados na verificação da normalidade de séries temporais. O procedimento empregado consiste na comparação gráfica dos quantis teóricos da distribuição normal com os quantis dos dados amostrais. A Figura 3 mostra a existência de uma relação não linear entre os quantis teóricos e empíricos, bastante acentuada nas caudas das distribuições, indicando caudas mais pesadas na distribuição empírica. Assim sendo, todos os testes rejeitaram a hipótese de normalidade da série analisada. 


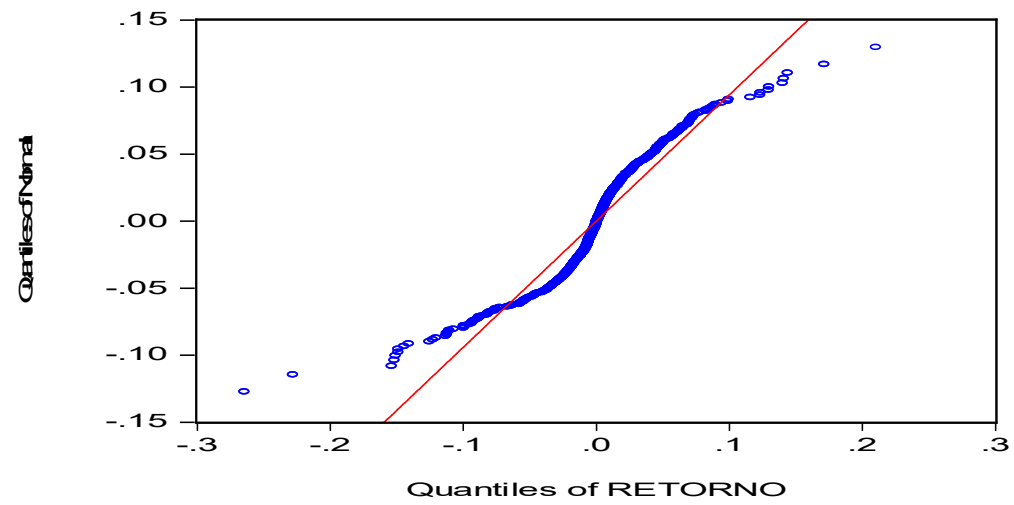

Figura 3: Plot Q-Q Retornos diários dos preços do etanol.

Antes de se efetivar a modelagem Box-Jenkins, é possível verificar formalmente se as séries dos retornos dos preços do etanol são estacionárias. Assim, os testes Dickey-Fuller Aumentado (ADF), PhillipsPerron (PP) e Kwiatkowski, Phillips, Schmidt e Shin (KPSS) com constante e com tendência, identificaram que as séries de retornos dos preços são estacionárias e não contém raízes unitárias, conforme se observa na Tabela 1.

Tabela 1: Teste de estacionaridade para a série dos retornos dos preços do etanol.

\begin{tabular}{|l|c|l|l|l|l|l|}
\hline Variável & ADF & $\begin{array}{l}\text { Valor Crítico } \\
(5 \%)\end{array}$ & $\begin{array}{l}\text { VPalor Crítico } \\
(5 \%)\end{array}$ & KPSS & $\begin{array}{l}\text { Valor } \\
(5 \%)\end{array}$ & \begin{tabular}{c} 
Crítico \\
\hline Etanol
\end{tabular} \\
\hline
\end{tabular}

Após a confirmação da estacionariedade, procedeu-se a escolha dos modelos ARMA para a estimação da equação da média da série dos retornos dos preços do etanol, visando eliminar o problema de correlação serial. Dentre os modelos analisados foi escolhido o modelo ARMA $(1,1)$, com base nos critérios de informação de Akaike (AIC) e Schwartz (SBC), melhor se ajustou ao comportamento da série, conforme apresentado na tabela 2.

Tabela 2: Critérios de seleção dos modelos ARMA

\begin{tabular}{|l|l|l|}
\hline \multicolumn{1}{|c|}{ Modelos } & AIC & SBC \\
\hline ARMA $(1,1)^{*}$ & $-3,9051$ & $-3,8896$ \\
\hline ARMA $(1,2)$ & $-3,9047$ & $-3,8892$ \\
\hline ARMA $(1,0)$ & $-3,8629$ & $-3,8525$ \\
\hline ARMA $(2,1)$ & $-3,9037$ & $-3,8882$ \\
\hline
\end{tabular}

$(*)$ melhor modelo ajustado.

Um teste de não linearidade pode ser necessário descrever as características da série histórica dos retornos dos preços do etanol. Assim, na tabela 3 mostra que os resultados apresentados indicam o efeito de não linearidade, ou seja, que as probabilidades são menores que 5\% ao nível de significância, implicando uma rejeição da hipótese nula de que a série dos retornos é linearmente dependente.

Tabela 3: Teste à independência temporal dos preços do etanol (BDS)

\begin{tabular}{|c|c|c|c|}
\hline Dimensão & Estatística BDS & Estatística Z & Probabilidade \\
\hline 2 & 0,0586 & 15,7909 & 0,0000 \\
\hline 3 & 0,1022 & 17,3128 & 0,0000 \\
\hline 4 & 0,1258 & 17,8749 & 0,0000 \\
\hline 5 & 0,1346 & 18,3172 & 0,0000 \\
\hline 6 & 0,1334 & 18,7882 & 0,0000 \\
\hline
\end{tabular}


O efeito assimétrico na volatilidade dos preços do etanol no Estado de São Paulo: uma aplicação do modelo Asymmetric power autoregressive

Antes de estimar o modelo APARCH, foi necessário realizar o teste $\mathrm{ARCH}$ para verificar se há ou não presença de heteroscedasticidade nos resíduos dos retornos, efetuou-se o teste LM (multiplicador de Lagrange) proposto por Engle (1982). Os valores do teste estão apresentados na Tabela 4. Observa-se que o teste mostra evidência contra a hipótese nula de não haver heteroscedasticidade condicional nos resíduos dos retornos dos preços do etanol.

Tabela 4: Teste ARCH.

\begin{tabular}{|l|l|l|l|l|}
\hline Lag & F stat & Prob & LM & Prob. \\
\hline 5 & 24,9807 & 0,0000 & 110,7981 & 0,0000 \\
\hline 10 & 15,4789 & 0,0000 & 134,0420 & 0,0000 \\
\hline
\end{tabular}

Assim sendo, foi calibrado o modelo de persistência e assimetria, no sentido de observar a dinâmica da volatilidade dos retornos dos preços do etanol. Foram utilizados três tipos de distribuições para os resíduos: normal (gaussiana), $t$ de student e GED (Generalized error distribution). A distribuição de erro generalizado obteve o melhor grau de ajuste de acordo com os critérios utilizados (AIC, SBC) (Tabela 5).

Tabela 5: Critérios de seleção entre as distribuições de erros do modelo.

\begin{tabular}{|l|l|l|l|}
\hline Modelo & Distribuição de erros & AIC & SBC \\
\hline \multirow{3}{*}{ ARMA (1,1) APARCH $(1,1)$} & Normal & $-4,3169$ & $-4,2808$ \\
& t-Student & $-4,4734$ & $-4,4321$ \\
& GED* & $-4,4894$ & $-4,4482$ \\
\hline
\end{tabular}

$(*)$ distribuição selecionada.

Analisando a Tabela 6 pode ser observado no modelo ARMA $(1,1)$ - APARCH $(1,1)$ - GED, que os coeficientes estimados são estatisticamente significativos ao nível de 5\%. A soma dos coeficientes $\alpha$ e $\beta$ é igual a 0,9684, indicando que um choque na série dos retornos dos preços do etanol terá efeito por muito tempo na volatilidade destes retornos. O coeficiente de persistência da volatilidade igual a 0,3709, indica que $37,1 \%$ de um choque gerado na série do retorno do etanol no tempo $t-1$ persistirá no tempo $t$. O AR(1) com o valor de 0,3199, estatisticamente significativo, representa o quanto o retorno do período $t-1$ influencia o retorno do período t. O MA(1) com o parâmetro de 0,1674 mostra o quanto as inovações (resíduos) do período anterior influenciam o retorno do período atual. Já o valor positivo de 0,0741 para o coeficiente ( $\gamma$ ) significa que choques negativos passados têm um impacto mais forte na volatilidade condicional do que choques positivos passados, indica a existência do efeito alavancagem. O coeficiente da potência assimétrica $\delta(1,3543)$, foi significativo ao nível de erro de $5 \%$ na série dos retornos dos preços do etanol.

Tabela 6: Resultado da estimação do modelo ARMA (1,1) - APARCH (1,1) - GED.

\begin{tabular}{|l|l|l|}
\hline Especificação & Coeficientes & p-valor \\
\hline Média Condicional & & \\
\hline AR (1) & 0,3199 & $0,0000^{*}$ \\
MA(1) & 0,1674 & $0,0027^{*}$ \\
\hline Variância Condicional & & \\
\hline$\alpha$ & 0,0020 & $0,0705^{* *}$ \\
\hline$\beta$ & 0,5975 & $0,0000^{*}$ \\
\hline$\gamma$ & 0,3709 & $0,0000^{*}$ \\
\hline$\delta$ & 0,0741 & $0,0445^{*}$ \\
\hline
\end{tabular}


O efeito assimétrico na volatilidade dos preços do etanol no Estado de São Paulo: uma aplicação do modelo Asymmetric power autoregressive

\begin{tabular}{|c|c|l|}
\hline \multicolumn{3}{|c|}{ Diagnósticos dos resíduos } \\
\hline Teste & Estatística & p-valor \\
\hline$Q^{2}(10)$ & 7.952 & $0,633^{*}$ \\
\hline$Q^{2}(15)$ & 14.424 & $0,494^{*}$ \\
\hline$Q^{2}(20)$ & 15.959 & $0,719^{*}$ \\
\hline LM-ARCH & 1.434 & $0,231^{*}$ \\
\hline
\end{tabular}

$\left.{ }^{*}\right)$ nível de significância de $5 \%$. $\left(^{* *}\right)$ nível de significância de $10 \%$

A análise dos resíduos do modelo ARMA $(1,1)$ - APARCH $(1,1)$ com distribuição GED, apresentada na tabela 6, percebe-se que o modelo utilizado apresenta adequabilidade, tanto para equação da média, quanto para a equação da variância, ao nível de $5 \%$ de significância, comprovado pelos testes de Ljung-Box (com 10, 15 e 20 defasagens) para os resíduos padronizados ao quadrado. Portanto, constata-se que os resíduos (inovações) padronizados são i.i.d. e que o efeito $\mathrm{ARCH}$ foi controlado, ou seja, que a equação da variância condicional é adequada.

\section{CONCLUSÕES}

No presente estudo, buscou-se estimar e analisar a persistência de choques e assimetria (efeito alavancagem) da volatilidade da série dos retornos dos preços do etanol. Os coeficientes estimados do modelo ARMA (1,1) - APARCH (1,1) - GED são estatisticamente significativos. A soma dos coeficientes $\alpha$ e $\beta$ é igual a 0,9684 , confirma que um choque na série dos retornos dos preços do etanol terá efeito por muito tempo na volatilidade destes retornos. Já o valor positivo obtido para o coeficiente $(\gamma)$ significa que choques negativos passados têm um impacto mais forte na volatilidade condicional do que choques positivos passados, indica a existência do efeito alavancagem. O coeficiente da potência assimétrica $\delta(1,3543)$, foi significativo ao nível de erro de $5 \%$ na série dos retornos dos preços.

O modelo utilizado apresenta forte avanço metodológico no tratamento dos retornos dos preços do etanol, servindo como ferramentas importantes no gerenciamento de risco pelos investidores.

\section{AGRADECIMENTOS}

À FAPERJ pela concessão da Bolsa de Pesquisa que possibilitou a realização desse artigo.

\section{REFERÊNCIAS}

ALMEIDA, N. M. C. G.; PEREIRA, P. L. V.. Mudança de regime em volatilidade: os modelos SWGARCH. In: ENCONTRO BRASILEIRO DE ECONOMETRIA, 21. Anais. 1999. p.39-58.

ARAUJO, B. V. F.; CAMARGOS, M. A.; PINHO, F. M..

Modelagem da volatilidade condicional incorporando o período não regular do pregão ao modelo APARCH. Revista Contabilidade \& Finanças, São Paulo, v.30, n.80, p.202-215, 2019. DOI: http://doi.org/10.1590/1808-057×201806100

BAIDYA, T. K. N.; COSTA, P. H. S.. Modelagem de séries financeiras brasileiras: previsão de preços de alguns ativos. Revista da Sobrapo, 1999.
BAILLIE, R. T.; BOLLERSLEV, T.. The message in daily exchange rates: a conditional variance tale. Journal of Business and Economic Statistics, v.7, p.297-305, 1989.

BALAKRISHNAN, N.; NEVZOROV, V. B.. A primer on statistical distribuitions. New Jersey, 2003.

BOLLERSLEV, T.; RAY, Y. C.; KENNETH, F. K.. ARCH modeling in finance: a review of the theory and empirical evidence. Journal of Econometrics, v.52, p.5-59, 1992.

BOLLERSLEV, T.. Generalized autoregressive conditional 
Heteroskedasticity. Journal of Econometrics, v.31, n.3, p.307-327, 1986

BOX, G. E.; TIAO, P. E.. Bayesian inference in statistical analysis. Reading: Addison-Wesley, 1973.

BOX, G. P.; JENKINS, G. M.. Time series analysis, forecasting and control. San Francisco: Holden-Day, 1976.

BROCK, W. A.; DECHERT, W. D.; SCHEINKMAN, J.. A test for independence based on the correlation dimension.

Madison: University of Wisconsin, SSRI Working Paper, 1987.

CHARLES, A.. The day-of-the-week effects on the volatility: the role of the asymmetry. European Journal of Operational Research, v.202, p.143-152, 2010.

COSTA JUNIOR, N. C. A.; CERETTA, P. S.. Influência de Evenos positivos e negativos sobre a volatilidade dos mercados na América Latina. Caderno de Pesquisa em Administração, v.1, n.10, 1999.

DICKEY, D. A.; FULLER, W.. A distribution of the estimators for autoregressive times series with unit root. Journal of the American Statistical Association, 1979.

DING, Z.; GRANGER, C. W. J.; ENGLE, R. F.. A long memory property of stock market returns and a new model. Journal of Empirical Finance, v.1, n.1, p.83-106, 1993.

DUARTE, J. A. M.; PINHEIRO, M. A.; HEIL, T. B. B.. Estimação da Volatilidade de Ativos e Índices Brasileiros. Resenha BM\&F, n.111, p.16-28, 1996.

ENGLE, R. F.. Autoregressive Conditional Heteroscedasticity with estimates of the variances of United Kingdon Inflation. Econometrica, v.50, n.4, p.987-1007, 1982.

ENGLE, R. F.; BOLLERSLEV, T.. Modelling the persistence of conditional variances. Econometric Review, v.5, p.1-50, 1986.

FREITAS, C. A.; SÁFADI, T.. Volatilidade dos retornos de commodities agropecuárias brasileiras: um teste utilizando o modelo APARCH. RESR, v.53, n.2, p.211-228, 2015.

GEWEKE, J.. Modeling the persistence of conditional variances: a comment. Econometric Review, v.5, p.57-61, 1986.

GLOSTEN, L. R.; JAGANNATHAN, R.; RUNKLE, D. E.. On the relation between the expected value and the volatility of the nominal excess returns on stocks. Journal of Finance, v.48, n.4, p.1779-1801, 1993.

HIGGINS, M.; BERA, A.. A class of nonlinear ARCH models. International Economic Review, v.33, p.137-158, 1992.

HSIEH, D.. Modeling heteroskedasticity in daily foreign exchange rates. Journal of Business and Economics Statistics, v.7, p.307-317, 1989.
ISSLER, J. V.. Estimating and forecasting the volatility of Brazilian finance series using ARCH Models. Revista de Econometria, v.19, n.1, p.5-56, 1999.

JARQUE, C.; BERA, A.. A test for normality of observations and regression residuals. International Statistical Review, v.55, p.163-172, 1987.

KWIATKOWSKI, D.; PHILLIPS, P. C. B.; SCHMIDT, P.; SHIN, Y.. Testing the null hypothesis of stationarity against the alternative of a unit root: How sure are we that economic time series have a unit root?. Journal of Econometrics, v.54, n.1, p.159-178, 1992.

LAURENT, S.. Analytical Derivates of the APARCH Model. Computational Economics, v.24, p.51-57, 2004.

MORAIS, I. A. C.; PORTUGAL, M. S.. Modelagem e previsão de volatilidade determinística e estocástica para a série do Ibovespa. Estudos Econômicos, v.29, n.3, p.303-34, 1999.

MOTA, B.; FERNANDES, M.. Desempenho de estimadores de volatilidade na Bolsa de Valores de São Paulo. Revista Brasileira de Economia, v.58, n.3, p.429-448, 2004.

NELSON, D. B.. Conditional heteroskedasticity in asset returns: a new approach. Econometrica, v.59, p.347-370, 1991.

NUGROHO, D. B.; SUZANTO, B.; PRATAMA, S. R.. Estimation of exchange rate volatility using APARCH-type models: a case study of Indonesia (2010-2015). Jurnal Ekonomi dan Studi Pembangunan, v.9, n.1, 2017. DOI: http://dx.doi.org/10.17977/um002v9i12017p065

PENTULA, S.. Modeling the persistence of conditional variances: a comment. Econometric Review, v.5, p.71-74, 1986.

PHILLIPS, P. C. B.; PERRON, P.. Testing for a unit root in time series regression. Biometrika, v.75, n.3, p.335-346, 1988.

SCHWERT, W.. Stock volatility and the crash of ' 87 . Review of Financial Studies, v.3, p.77-102, 1990.

SILVA, C. A. G.. Uma análise empírica da volatilidade do retorno da ação da Petrobrás. In: ENCONTRO DE MODELAGEM COMPUTACIONAL, 10. Anais. Nova Friburgo: UERJ, 2006.

SUBBOTIN, M. T.. On the law of frequency of error. Matematicheskii Sbornik, v.31, p.296-301, 1923.

TAYLOR, S.. Modeling financial time series. New York: Wiley, 1986.

TSAY, R. S.. Analysis of financial time series. Series in Probability and Statistics. New Jersey: Wiley, 2010.

ZAKOIAN, J. M.. Threshold Heteroskedascity Models. Journal of Economic Dynamics and Control, v.18, p.931-955, 1994.

A CBPC - Companhia Brasileira de Produção Científica (CNPJ: 11.221.422/0001-03) detém os direitos materiais desta publicação. Os direitos referem-se à publicação do trabalho em qualquer parte do mundo, incluindo os direitos às renovações, expansões e disseminações da contribuição, bem como outros direitos subsidiários. Todos os trabalhos publicados eletronicamente poderão posteriormente ser publicados em coletâneas impressas sob coordenação da Sustenere Publishing, da Companhia Brasileira de Produção Científica e seus parceiros autorizados. Os (as) autores (as) posteriormente ser publicados em coletâneas impressas sob coordenação da Sustenere Publishing, da Companhia Brasileira de Produção Científica e seus para
preservam os direitos autorais, mas não têm permissão para a publicação da contribuição em outro meio, impresso ou digital, em português ou em tradução. 